\title{
Is sham cTBS real cTBS? The effect on EEG dynamics
}

\section{Alexander Opitz ${ }^{1,2,3 *+}$, Wynn Legon ${ }^{4 \dagger}$, Jerel Mueller ${ }^{5}$, Aaron Barbour ${ }^{6}$, Walter Paulus ${ }^{1}$ and William J. Tyler ${ }^{7}$}

${ }^{1}$ Department of Clinical Neurophysiology, Georg-August-University, Göttingen, Germany

${ }^{2}$ Center for Biomedical Imaging and Neuromodulation, Nathan Kline Institute for Psychiatric Research, Orangeburg, NY, USA

${ }^{3}$ Center for the Developing Brain, Child Mind Institute, New York, NY, USA

${ }^{4}$ Department of Physical Medicine and Rehabilitation, University of Minnesota, Minneapolis, MN, USA

${ }^{5}$ School of Biomedical Engineering and Sciences, Virginia Tech, Blacksburg, VA, USA

${ }^{6}$ Virginia Tech Carilion Research Institute, Roanoke, VA, USA

' School of Biological and Health Systems Engineering, Arizona State University, Tempe, AZ, USA

\section{Edited by:}

Srikantan S. Nagarajan, University of California, San Francisco, USA

\section{Reviewed by:}

Shozo Tobimatsu, Kyushu

University, Japan

Theodore Zanto, University of

California, San Francisco, USA

${ }^{*}$ Correspondence:

Alexander Opitz, Center for

Biomedical Imaging and

Neuromodulation, Nathan Kline

Institute for Psychiatric Research,

140 Old Orangeburg Road Bldg.35,

Orangeburg, NY 10962, USA

e-mail: aopitz@nki.rfmh.org

${ }^{\dagger}$ Co-first author.
Increasing sensitivity of modern evaluation tools allows for the study of weaker electric stimulation effects on neural populations. In the current study we examined the effects of sham continuous theta burst (cTBS) transcranial magnetic stimulation to the left dorsolateral prefrontal cortex (DLPFC) upon somatosensory evoked potentials (SEP) and frontal-parietal phase coupling of alpha and beta bands. Sham TMS results in an induced electric field amplitude roughly 5\% that of real TMS with a similar spatial extent in cortex. Both real and sham cTBS reduced the amplitude of the frontal P14-N30 SEP and increased local phase coupling in the alpha-beta frequency bands of left frontal cortex. In addition, both sham and real cTBS increased frontal-parietal phase coupling in the alpha-beta bands concomitant with an increase in amplitude of parietal P50-N70 complex. These data suggest that weak electric fields from sham cTBS can affect both local and downstream neuronal circuits, though in a different manner than high strength TMS.

Keywords: TMS, EEG, electric field, sham TMS, DLPFC, evoked potentials, somatosensory

\section{INTRODUCTION}

Non-invasive neuromodulation methods rely on effects produced by a broad range of electric field strengths ranging from $100 \mathrm{mV} / \mathrm{mm}$ for transcranial magnetic stimulation (TMS; Miranda et al., 2007; Salvador et al., 2011) to only a fraction of $1 \mathrm{mV} / \mathrm{mm}$ for transcranial direct current stimulation or transcranial alternating current stimulation (tDCS/tACS; Datta et al., 2009; Salvador et al., 2010; Miranda et al., 2013). It has recently been shown that low frequency $(<8 \mathrm{~Hz})$ extracellular electric fields smaller than $1 \mathrm{mV} / \mathrm{mm}$ can cause neuronal entrainment by ephaptic coupling in rat cortical pyramidal neurons (Anastassiou et al., 2011). Additional evidence has demonstrated that electric fields lower than $1 \mathrm{mV} / \mathrm{mm}$ can produce effects in hippocampal slices for alternating current (AC) fields having a frequency range from 10 to $100 \mathrm{~Hz}$ (Deans et al., 2007) and low-frequency pulsed electric fields (Francis et al., 2003), as well as for direct current (DC) fields $<10$ and $<4 \mathrm{mV} / \mathrm{mm}$ (Bikson et al., 2004; Radman et al., 2007). Furthermore, neocortical activity can also be influenced by low-frequency sinusoidal electric fields $<0.5 \mathrm{mV} / \mathrm{mm}$ (Frohlich and McCormick, 2010; Ozen et al., 2010). Collectively, it is important to realize that weak electric fields from both DC (Nitsche and Paulus, 2000) and AC stimulation (Moliadze et al., 2010) have been shown to transiently alter cortical excitability in humans.

Continuous theta burst stimulation (cTBS) is a repetitive TMS protocol that can induce cortical excitability changes in a short stimulation period (Huang et al., 2005). cTBS typically operates at $70 \%$ of individual motor threshold which corresponds to electric field strengths in the range of $50-80 \mathrm{mV} / \mathrm{mm}$. This range of electric field strength is approximately two orders of magnitude higher than what is minimally needed to cause an effect on neurons (Francis et al., 2003). Despite this, the effects of weak electric fields induced by TMS have not been extensively studied. These issues are especially important for TMS studies applying sham protocols. TMS shamming has taken many forms over the years, though currently accepted approaches use coils with magnetic shields that reduce the magnetic field strength and attenuate the induced electric field by about $80-95 \%$ depending on the coil type and manufacturer. While it is generally assumed that these sham coils have no neuronal effect (Duecker and Sack, 2013) the induced electric fields are indeed on a range that could affect cortical excitability.

In the present study we investigated how weak electric fields induced by sham TMS over the left dorsolateral prefrontal cortex (DLPFC) exert effects upon local and functionally connected neuronal circuitry using electroencephalographic (EEG) measures of phase connectivity and amplitude of somatosensory evoked potentials (SEPs). To better assess the effect of sham TMS, we included a biologically inert "sham-sham" stimulation condition. In addition, we used computational modeling to evaluate the electric field distribution induced by real and sham TMS. Our observations demonstrate that weak electric fields generated by sham TMS can exert direct neural effects that differ from real TMS as well as the sham-sham condition. 


\section{MATERIALS AND METHODS PARTICIPANTS}

Fourteen participants (7 male, age 19-61, mean $34.3 \pm 16.5$ years) were included in the first part of the experiment testing cTBS versus sham stimulation. Fourteen participants (10 male, age 18-38, mean $25.7 \pm 6.7$ years) were included in the second portion of the experiment testing the effect of a biologically inert (sham-sham) stimulation versus cTBS and sham stimulation. Two participants who took part in the first experiment also took part in the second part of the experiment. All participants provided written informed consent to voluntarily participate in the study and received remuneration for participation. None of the participants reported current drug use (prescription or otherwise) or a history of neurological impairment and all were self-report right hand dominant. All procedures were approved by the Institutional Review Board at Virginia Tech.

\section{TMS}

For the first portion of experiment 1, participants underwent both cTBS and sham TMS protocols on separate days separated by at least $48 \mathrm{~h}$. Both cTBS and sham stimulation was performed with a MagPro X100 Stimulator (MagVenture, Inc., Atlanta, GA, USA) and Cool-B65-A-P-Butterfly-Coil (2 layers of 5 windings at each wing, winding height $12 \mathrm{~mm}$, inner diameter $35 \mathrm{~mm}$, outer diameter $75 \mathrm{~mm}$ ). The coil was positioned over the international 10-20 electrode site F3 with the coil handle angled $45^{\circ}$ outward from midline. A continuous theta burst (cTBS) repetitive protocol was used according to Huang et al. (2005) at 70\% of participants' resting motor threshold. Briefly, cTBS consists of 3 single biphasic pulses separated by $0.02 \mathrm{~s}(50 \mathrm{~Hz})$ repeated every $0.2 \mathrm{~s}(5 \mathrm{~Hz})$ for a total of 600 pulses delivered in $40 \mathrm{~s}$. The mean resting motor threshold was $55 \pm 6 \%$ of maximum stimulator output. Sham stimulation was performed with the identical parameters and intensity using the "sham" side of the coil. The sham side of the coil uses a mu metal plate for reduction of the magnetic field from the TMS coil. For both real and sham stimulation, participants wore surface electrodes (Neuroline 710, Ambu Inc. MD, USA) separated by $5 \mathrm{~cm}$, placed on the forehead $3 \mathrm{~cm}$ above the left eye. Low current electrical stimulation was delivered through these electrodes in unison with the TMS to help mask and mimic TMS sensation and improve shamming according to recommended procedures. The intensity of the electric pulses was individually set for each participant to a " 6 out of 10 " on a discomfort scale where 0 was "no feeling," 5 "slightly uncomfortable" and 10 was "painful cannot continue." All participants tolerated the real and sham stimulation well.

Individual motor thresholds were determined using a C-B60 coil (figure- 8 coil, 2 layers of 5 windings at each wing, winding height $11 \mathrm{~mm}$, inner diameter $35 \mathrm{~mm}$, outer diameter $75 \mathrm{~mm}$ ). The coil was placed on the left hemisphere over the motor strip with handle position at $45^{\circ}$ outward from midline. The coil was moved in $1 \mathrm{~cm}$ increments and single pulses were delivered every $5 \mathrm{~s}$ and stimulator intensity adjusted until half of the total pulses resulted in a small, but noticeable twitch of the right thumb.

Collection for the second portion (sham-sham) of the experiment was identical to that described above, except that shamsham stimulation consisted of placing a custom made biologically inert "coil" on the head. This coil was made from wood and metal piping to convey the same physical (pressure) sensation upon the head as both the cTBS and sham stimulation protocols. Participants were unable to view the coil or the stimulation procedure. Participants underwent motor threshold testing as above and were outfitted with the identical forehead electrodes to mimic electrical stimulation as in the first portion of the experiment and were additionally outfitted with earphones that played a recorded audio file of the cTBS protocol to mimic auditory stimulation from both cTBS and sham stimulation procedures.

\section{EEG}

Electroencephalographic (EEG) data were acquired using a DC amplifier (BrainAmp MR Plus, Brain Products GmbH Gilching Germany) with an acticap 64 channel cap (Brain Products $\mathrm{GmbH})$, referenced to the average of 64 channels.

Data were sampled at $1000 \mathrm{~Hz}$ and online filtered at DC$250 \mathrm{~Hz}$. Impedances of all electrodes were confirmed to be $<5 \mathrm{k} \Omega$. Somatosensory evoked potentials (SEPs) were derived from electrical stimulation of the median nerve of the right wrist using square wave pulses of $0.5 \mathrm{~ms}$ duration (GRASS SD9 stimulator, West Warwick, Rhode Island, USA) delivered through a surface bar electrode ( $2 \mathrm{~cm}$ electrode spacing), anode distal, fixed to the wrist using tape. The intensity of the pulse was adjusted until a slight but noticeable thumb twitch was achieved. Electrical pulses were delivered at an interstimulus interval of $1.2 \mathrm{~s}$ with a positive randomization of $1.5 \mathrm{~s}$. A total of 250 stimuli were delivered for each session. Stimulation intensity was monitored via surface electromyography (EMG) of the thumb using a SX230 EMG sensor ( $1 \mathrm{~cm}$ diameter, $2 \mathrm{~cm}$ spacing) and K800 amplifier (Biometrics Ltd, Ladysmith Virginia USA) and sampled at $100 \mathrm{~Hz}$ using Spike2 version 7.08a software (CED, UK) and stored on a computer for later analysis. EEG data were collected prior to the cTBS/Sham intervention (Pre) and at two time points post.

cTBS/Sham stimulation (Post1: 5-7 min; and Post2: $25 \mathrm{~min}$ ). During median nerve stimulation participants were required to fixate on a cross on a computer screen placed in front of them. Total testing time for each EEG recording session was roughly $6 \mathrm{~min}$.

\section{EEG - weighted phase lag index}

EEG data were processed using custom Matlab scripts (Matlab The Mathworks, Inc., Natick, MA), EEGLAB (Delorme and Makeig, 2004) and Fieldtrip (Vinck et al., 2011). For all EEG analyses we focused on the pre stimulation and $25 \mathrm{~min}$ post stimulation data to evaluate the effect of the sham stimulation. This was chosen as there was a gradual buildup over time (see Supplementary Figure 1 for an exemplary illustration) with maximal effect in Post2. In the following analysis we refer to the Post 2 recording as Post condition. Data were re-referenced to the average of 64 channels, band-pass filtered $(1-90 \mathrm{~Hz})$ and notch filtered at $60 \mathrm{~Hz}$. Data were inspected for artifacts using automatic rejection criteria of absolute peak-to-peak amplitude of $100 \mu \mathrm{V}$ and $80 \mu \mathrm{V} / \mathrm{ms}$ using channels Fp1, Fp2, Af3, Af4, F3, and Cp3. Data were epoched around median nerve stimulus onset $(-200$ to $500 \mathrm{~ms})$ and baseline corrected $(-200$ to $0 \mathrm{~ms}$ ). To assess the effect of both cTBS, sham and sham-sham TMS on local and downstream connected neuronal regions, phase synchronization between local prefrontal areas as well as 
long range prefrontal and somatosensory brain areas was computed using the weighted phase lag index (WPLI) (Vinck et al., 2011) which is unaffected by volume conduction. WPLI was computed between local channel pairs F3-F1, F3-F5, F3-FC1, F3-FC3, and F3-FC5 as well as F3-CP3, F3-CP4, F3-F4, and
CP3-CP4. Differences in phase synchronization were computed between Post and Pre stimulation for all three conditions (cTBS, sham and sham-sham). Statistical analysis of the Post-Pre WPLI was performed using non-parametric cluster threshold analysis (Maris and Oostenveld, 2007) with 10,000 repetitions, initial
A

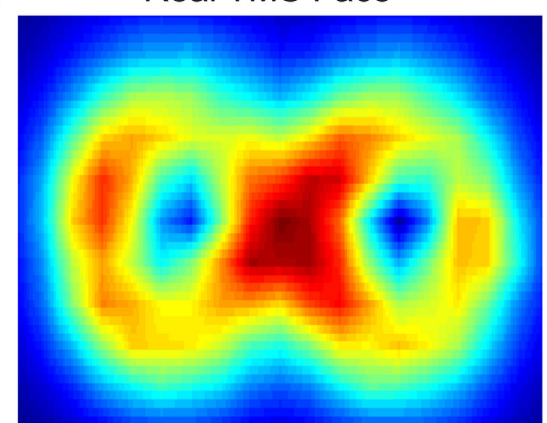

Real TMS Face
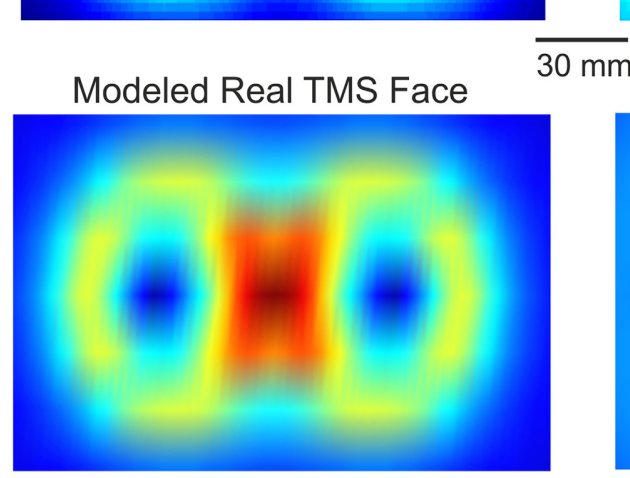

0

B

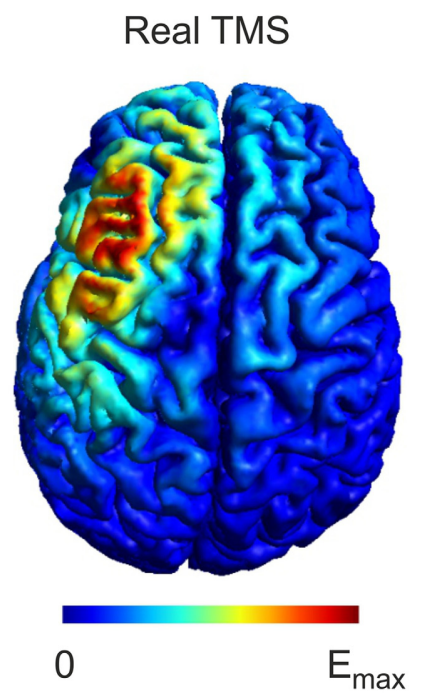

FIGURE 1 | Real and Sham TMS fields. (A) Distributions of the magnetic vector potential for the real and sham side of the TMS coil. The upper panels illustrate measured values for both real and sham faces of the coil while the lower panels illustrate the modeled field distribution. The magnetic vector potential for the real side is about 20 times stronger than for the sham side. The coil models faithfully capture

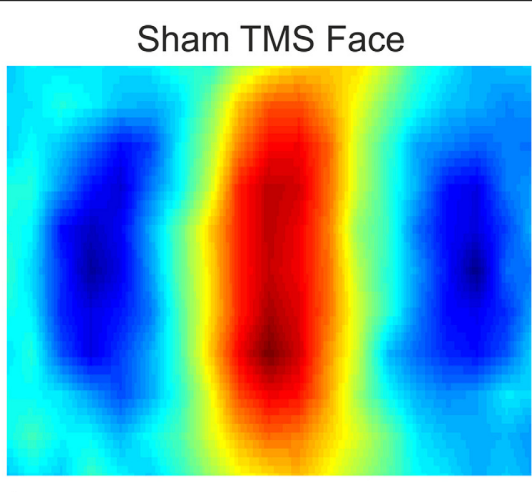

Modeled Sham TMS Face

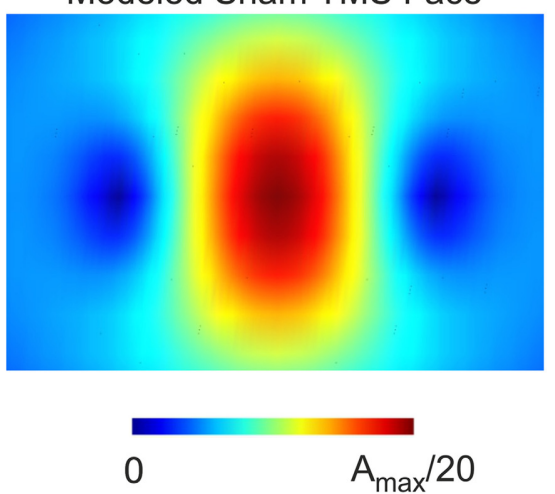

Sham TMS

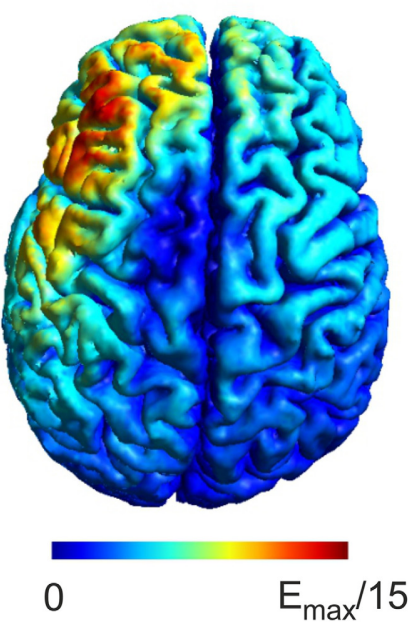

the measured field distribution as illustrated. (B) The simulated electric field distributions generated by real and sham cTBS of the left DLPFC. The electric field strength generated by real TMS is about 15 times stronger than the electric field strength produced by sham TMS. The spatial distributions of the electric field generated by real and sham TMS were strikingly similar to one another. 
cluster threshold $p=0.05$ for the time points between -100 and $400 \mathrm{~ms}$ and frequency bands $7-13$ and $14-30 \mathrm{~Hz}$ and FDR correction (Benjamini-Hochberg, false discovery rate $q=0.05$ ) for number of channel combinations and conditions. This procedure was conducted separately for channel combinations starting from channel CP3 (3 combinations $\times 3$ conditions) and channel combinations starting from channel F3 (7 combinations $\times 3$ conditions). For cTBS vs sham a dependent $t$-test was conducted, and for cTBS vs. sham-sham, as well as sham vs. sham-sham, an independent $t$-test was conducted. To control for potential existing between group differences in EEG, we also tested for possible baseline differences between groups for the three Pre conditions using the same procedure as for the Post-Pre comparisons.

\section{EEG - somatosensory evoked potentials}

Data were preprocessed as above and epoched around median nerve stimulation onset ( -100 to $300 \mathrm{~ms}$ ) and baseline corrected $(-100$ to $0 \mathrm{~ms})$. Peak to peak amplitudes were quantified from channel F3 and CP3. For channel F3 potentials included P14N30, N30-P45, P45-N60, N60-P80, and P80-N100. For channel CP3, potentials included P14-N20, N20-P27, N27-N33, N33-P50, P50-N70, N70-P100, and P100-N140. Potentials were quantified for each participant for each stimulation procedure (cTBS, sham, sham-sham) for both the Pre and Post conditions. A One-Way independent samples analysis of variance (ANOVA) was conducted for each of the potentials from channel F3 and CP3 on the Post-Pre data with factors (cTBS, sham, sham-sham). Significant

\section{A F3 - CP3 Connectivity}

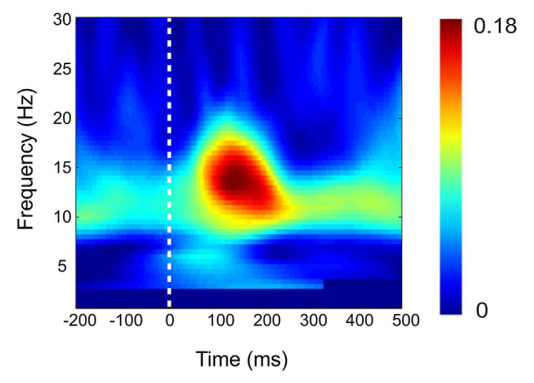

B

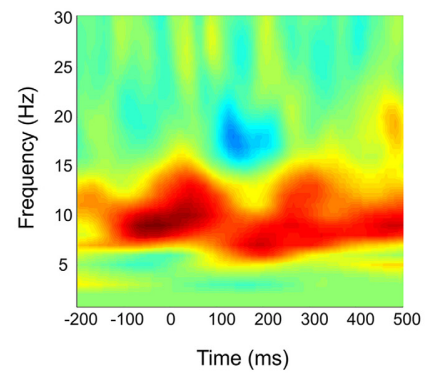

C

Real vs Sham

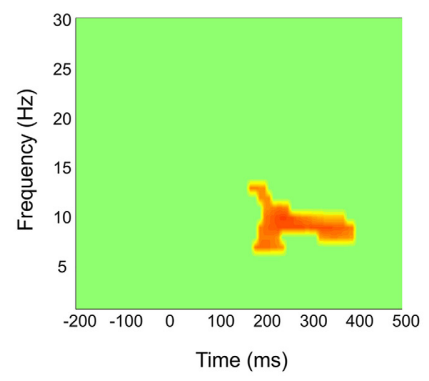

Sham

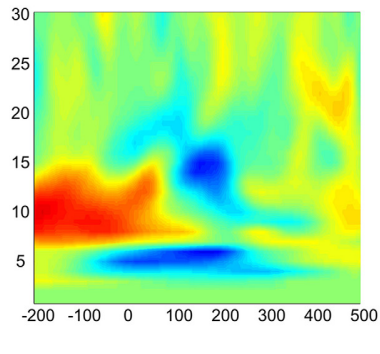

Real vs ShamSham

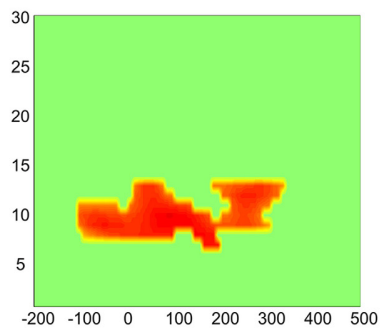

ShamSham

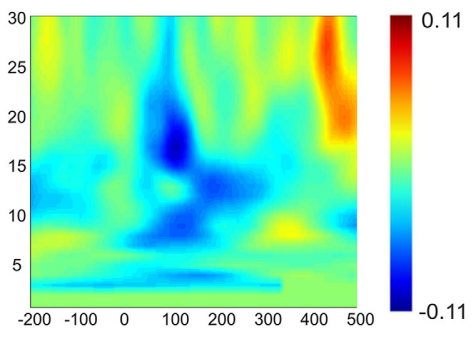

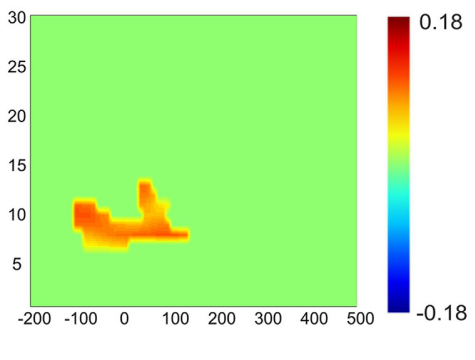

FIGURE 2 | Quantification of changes in cortical EEG connectivity. (A) The baseline weighted phase lag index (WPLI) for the F3-CP3 channel pair during median nerve stimulation is shown. The dashed lines indicate the onset of the median nerve stimulus. An increase in connectivity in the high alpha, low beta range is visible after the stimulus onset. (B) Connectivity changes (Post-Pre) are shown for real, sham and sham-sham stimulation.
Differential effects on connectivity changes are visible depending on stimulation condition. (C) Significant differences between the connectivity changes in real vs. sham, real vs. sham-sham, as well as sham vs. sham-sham are shown. Strongest effects were present for real vs. sham-sham, with a strong increase in the WPLI in the alpha and low beta band in the real condition. 
$(p<0.05) F$-tests were evaluated with post-hoc Tukey-Kramer tests.

\section{MAGNETIC FIELD MAPPING}

The magnetic field of the real and sham side of the TMS coil was measured using a custom built magnetic probe made of two rectangular shaped windings of wires $\left(1 \mathrm{~cm}^{2}\right.$ surface area) that were oriented perpendicularly to each other. A grid of $19 \times 19$ points ( $1 \mathrm{~cm}$ spacing) was placed directly on the coil face to measure the magnetic field induced voltages in $\mathrm{x}$ and $\mathrm{y}$ direction for both the real and sham face of the TMS coil. A computational model of the TMS coil that reproduces the spatial pattern of the measured magnetic field was constructed using a dipole approximation (Thielscher and Kammer, 2004). Small magnetic dipoles are positioned at the coil windings and the total magnetic vector potential is obtained by summing up individual magnetic dipoles. For the sham coil side, the effect of the magnetic shield was mimicked by increasing the distance of the coil to the measurement plane in the computational model such that the computational and measurement data match each other.

\section{ELECTRIC FIELD SIMULATIONS}

Electric field simulations were performed for both the active and sham side of the TMS coil. A finite element model of the head for one subject was constructed using Simnibs (Windhoff et al., 2013). From T1- and T2- weighted MR images five different tissue types WM, GM, CSF, skin and skull were segmented and a FEM model consisting of ca. 1.7 million tetrahedral elements was constructed. TMS coil position and orientation over the left DLPFC was recorded using a neuronavigation system (Visor 2, ANT). For more detailed methods about the FEM simulations see (Opitz et al., 2011, 2013; Windhoff et al., 2013).

\section{RESULTS}

\section{MAGNETIC FIELD MEASUREMENTS AND ELECTRIC FIELD SIMULATIONS}

The sham side of the TMS coil produced a magnetic field maximum approximately 20-fold less than the real side (Figure 1A). The field distribution of the real side has a shape typical of figure8 coils with highest field strength at the coil center and moderate field strengths at the wings of the coil. The sham side of the coil exhibited a broad area of high field strength only in the center region of the coil. The computational model of the TMS coil was able to accurately capture the general distribution of the measured magnetic field (Figure 1A). The root mean square error between the measured and modeled magnetic field was 7.7 and $7.5 \%$ in the central region of the coil $(1 \times 1 \mathrm{~cm})$ for the real and sham sides respectively. In one circumscribed region at the coil wing the error was up to $50 \%$ which was likely due to a small asymmetry in the coil with regard to the measurement plane. However, as the electric field distribution is mainly driven by the center of the coil this was unlikely to significantly influence the accuracy of the electric field simulations.

While the maximum magnetic field of the sham coil side was reduced 20 -fold, the maximum induced electric field was only reduced 15-fold (Figure 1B). Furthermore, the electric field distribution was broader for the sham condition. The broader distribution of the sham side is due to the smearing effect of the magnetic shield, which defocusses as well as reduces the magnetic and the electric field.

\section{STIMULATION EFFECTS ON PHASE SYNCHRONY}

Based on the observations described above, we next examined the functional connectivity between local frontal electrodes as well as downstream connections using the weighted phase lag index (WPLI) as a measure of phase synchronization. Of special interest was the effect between the stimulation site F3 and the localization site of parietal somatosensory evoked potentials recorded from electrode site CP3 based upon previous research demonstrating this connection (Yamaguchi and Knight, 1990). Under baseline, the WPLI showed a significant increase in alpha and beta $(10-20 \mathrm{~Hz})$ coupling between CP3 and F3 immediately after median nerve stimulation $(p<0.025)$ (Figure 2A). This CP3-F3 phase synchronization was considerably stronger than local F3 channel pairs $(p<0.025)$ (Figure 3). After real cTBS the WPLI showed a broad increase for alpha and beta frequencies $(p<$ 0.025) (Figure 2B). After sham cTBS we observed a decrease in beta as well as theta activity occurring at 100-200 ms (Figure 2B). For sham-sham a broad decrease in the WPLI after median

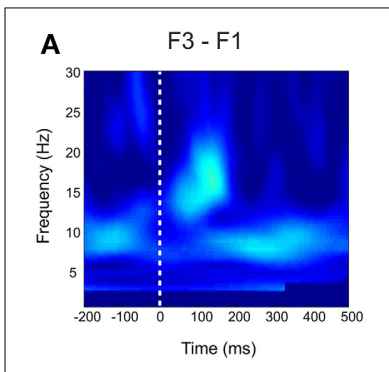

C

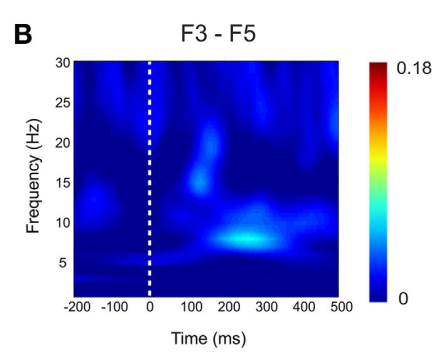

D
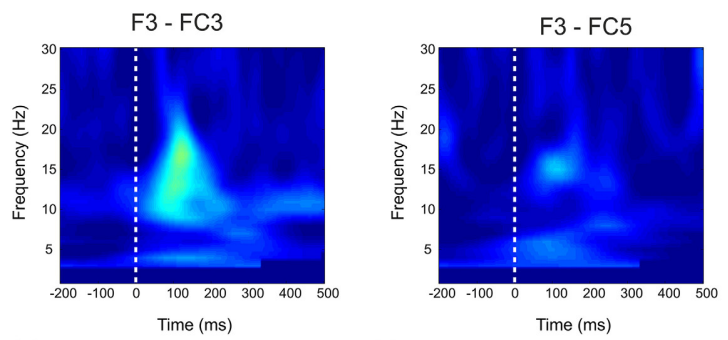

E

F3 - F4

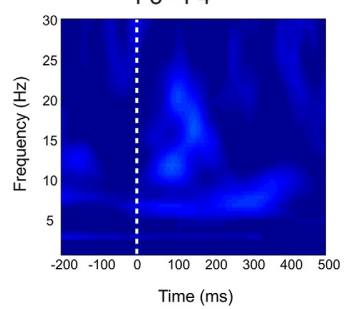

$\mathbf{F}$

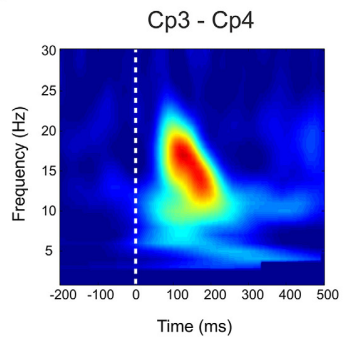

FIGURE 3 | Baseline Connectivity for local and interhemispheric connections. Baseline weighted phase lag index (WPLI) during median nerve stimulation for local channel connections (A-D) and interhemispheric connections (E-F). 
nerve onset could be seen in the Post-condition compared to baseline (Figure 2B). Individual comparisons showed significant effects between all conditions (Figure 2C). A strong enhancement of phase coupling in the alpha-beta frequency range (7$14 \mathrm{~Hz})$ was found comparing cTBS with sham-sham $(p<0.0083)$ (Figure 3C middle panel). Similar effects, yet for shorter time windows, were found for the cTBS vs. sham and sham vs. shamsham comparisons (Figure 2C). Most importantly, sham cTBS showed significant increases in phase coupling in the alpha-beta frequency compared to the sham-sham condition $(p<0.0083)$. These data indicate that both cTBS and sham cTBS can induce lasting changes amongst cortical networks which differ from a biologically inert control condition. There were no significant differences in the baseline connectivity between stimulation conditions $(p>0.05)$.
For the local channel pair F3-FC1, a similar effect as for F3CP3 was found for real vs sham-sham $(p<0.0083)$ (Figure 4), however no effects were found for the other comparisons. We did not observe any stimulation related changes in crosshemispheric phase synchronization between F3-F4 and CP3-CP4 despite strong intrinsic coupling between $\mathrm{CP} 3$ and $\mathrm{CP} 4$ in the alpha-beta frequency range (Figure 3). Interestingly, stimulation effects were apparent between F4-CP3 and F3-CP4 (Figures 5, 6) with a significant difference $(p<0.0083)$ between real and sham-sham.

\section{SOMATOSENSORY EVOKED POTENTIALS}

Somatosensory evoked potential peak to peak amplitudes were quantified from channel $\mathrm{F} 3$ and $\mathrm{CP} 3$. For channel $\mathrm{CP} 3$ a significant effect was found for the N70-P50 SEP complex

\section{A F3 - FC1 Connectivity}

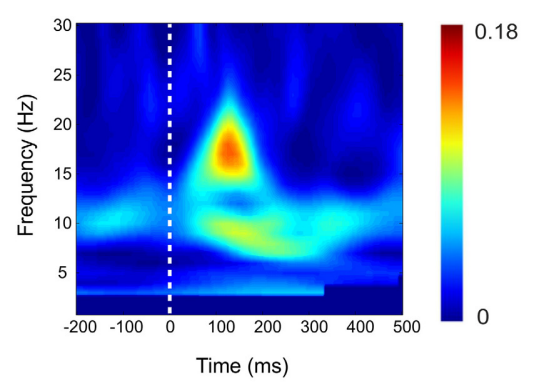

B

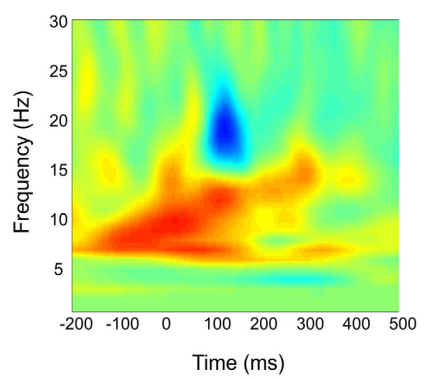

C

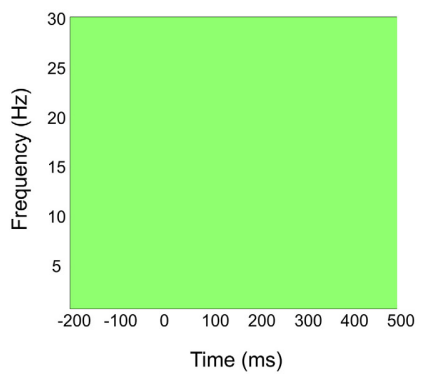

Sham

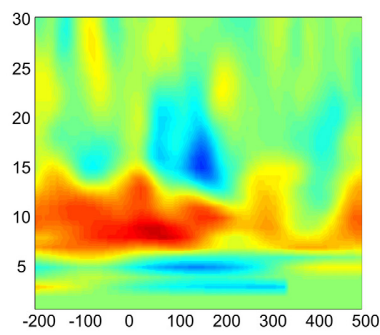

Real vs ShamSham

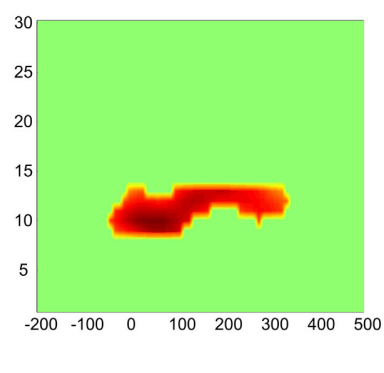

ShamSham

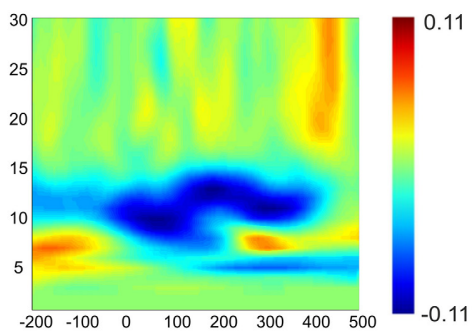

Sham vs ShamSham

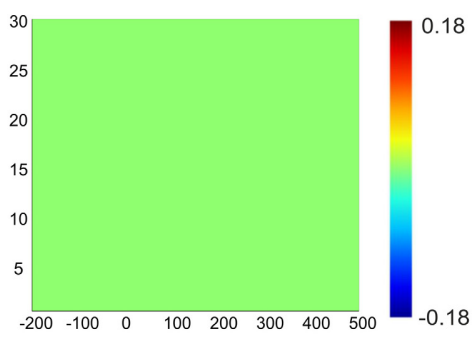

FIGURE 4 | Connectivity changes in F3-FC1. (A) Baseline weighted phase lag index (WPLI) during median nerve stimulation. (B) Connectivity changes (Post-Pre) for real, sham and sham-sham stimulation. (C) Significant differences between the connectivity changes in real vs. sham, real vs. sham-sham and sham vs. sham-sham. 


\section{A F4 - CP3 Connectivity}

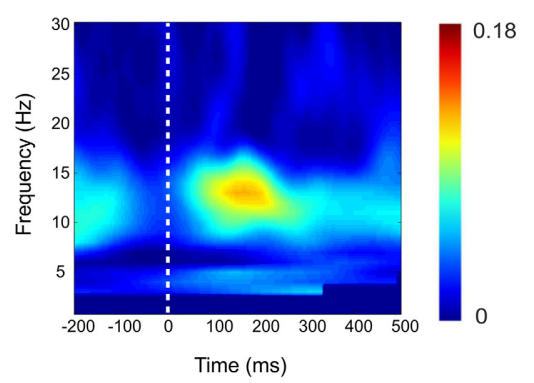

B

Real

Sham

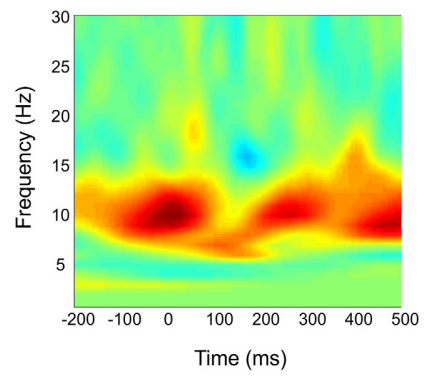

C

Real vs Sham

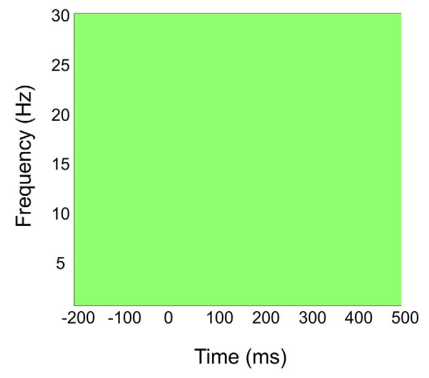

Real vs ShamSham

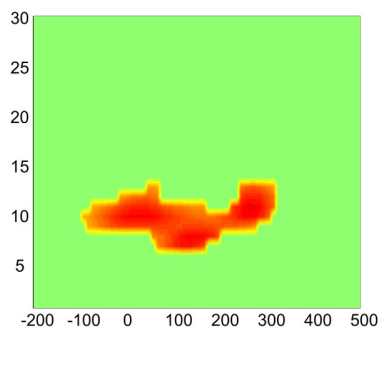

ShamSham

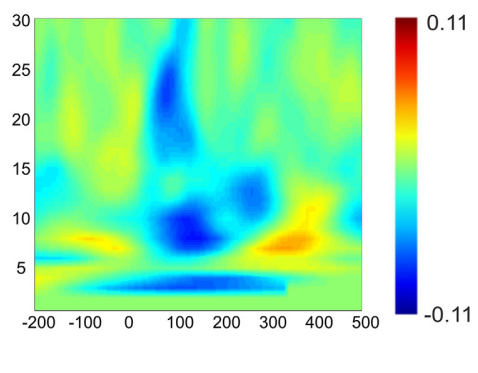

Sham vs ShamSham

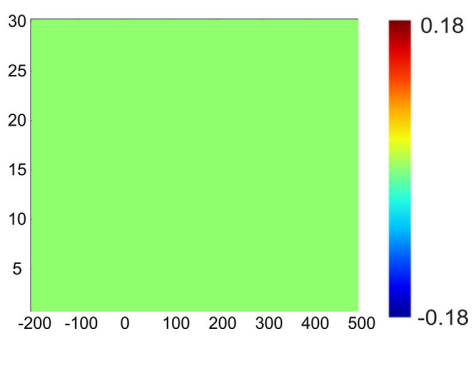

FIGURE 5 | Connectivity changes in F4-CP3. (A) Baseline weighted phase lag index (WPLI) during median nerve stimulation. (B) Connectivity changes (Post-Pre) for real, sham and sham-sham stimulation. (C) Significant differences between the connectivity changes in real vs. sham, real vs. sham-sham and sham vs. sham-sham.
$\left[F_{(2,39)}=3.27, p=0.0491\right]$. A post-hoc Tukey-Kramer test revealed a significant increase $(p<0.05)$ in amplitude of the N70-P50 for cTBS compared to sham-sham only (Figure 7A). No statistically significant differences were found for any of the other quantified potentials (Table 1). For channel F3 a significant decrease in amplitude was found for the P14N30 potential $\left[F_{(2,39)}=4.04, p=0.0258\right]$. Post-hoc TukeyKramer tests revealed a significant decrease in amplitude of the peak to peak P14-N30 potential for cTBS compared to sham-sham $(p<0.05)$, and for a significant decrease in amplitude for sham compared to sham-sham $(p<0.05)$ (Figure 7B and Table 1). No statistically significant differences were found for any of the other potentials quantified from channel F3 (Table 1).

\section{DISCUSSION}

We investigated the effects of weak electric fields induced by sham cTBS to the left DLPFC on EEG phase connectivity and SEP amplitudes. Results demonstrate that both real and sham cTBS over the left DLPFC increase both local and down-stream phasecoupling in the alpha-beta $(7-14 \mathrm{~Hz})$ frequency range and both decrease amplitude of frontal SEPs but only real cTBS to significantly increase amplitude of parietal SEPs. The results of this study provide evidence that low electric field strengths from short duration cTBS can have an influence on local and downstream cortical activity.

tACS and tDCS produce electric fields in the brain roughly an order of magnitude less than sham TMS as used here. It should not be too surprising then to find effects upon somatosensory 


\section{A F3 - CP4 Connectivity}

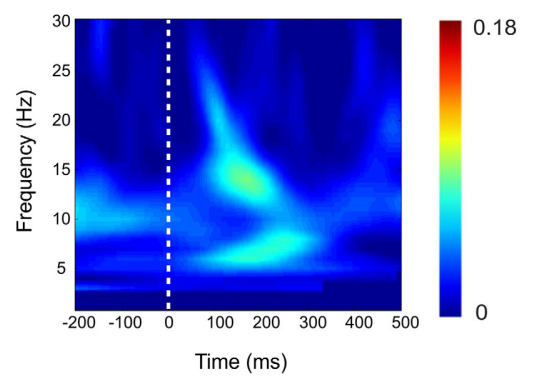

B

Real

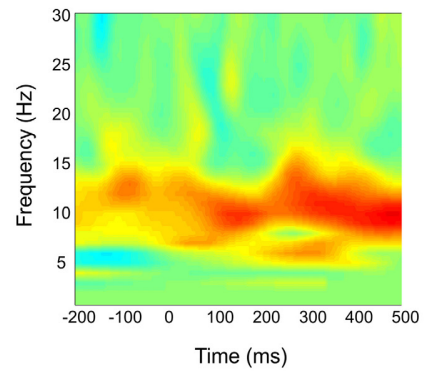

C

Real vs Sham

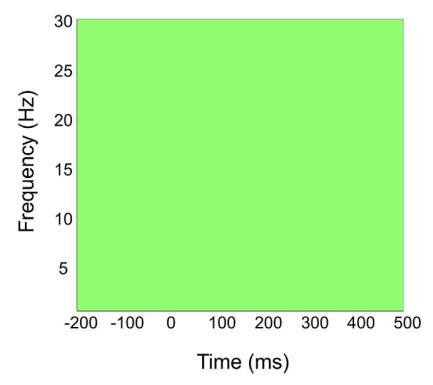

Sham

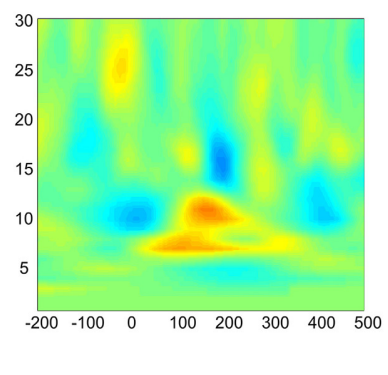

Real vs ShamSham

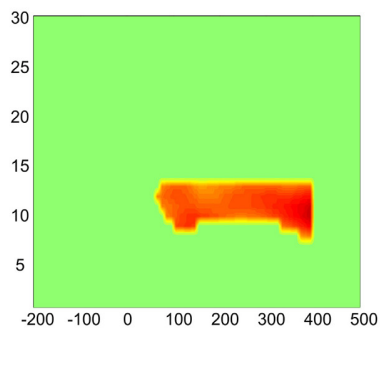

ShamSham

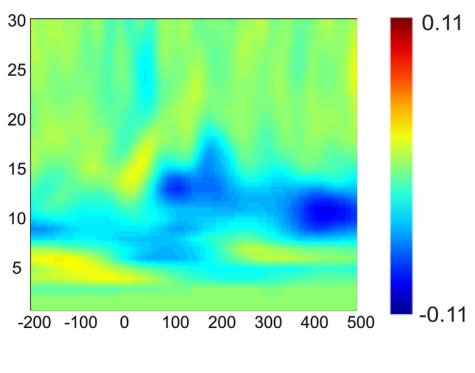

Sham vs ShamSham

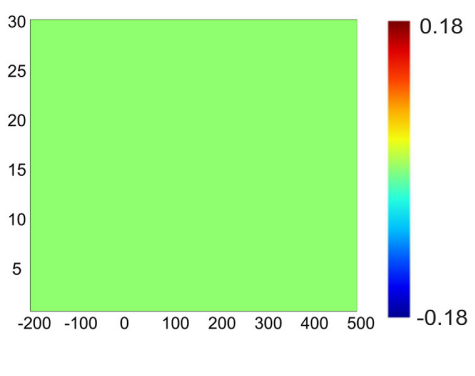

FIGURE 6 | Connectivity changes in F3-CP4. (A) Baseline weighted phase lag index (WPLI) during median nerve stimulation. (B)

Connectivity changes (Post-Pre) for real, sham and sham-sham stimulation. (C) Significant differences between the connectivity changes in real vs. sham, real vs. sham-sham and sham vs. sham-sham. activity from sham stimulation given that tACS (Feurra et al., 2011) and tDCS (Matsunaga et al., 2004; Dieckhofer et al., 2006) also affects somatosensory processing. Here, weak stimulation was delivered over the frontal cortex, yet changes were recorded in SEP potentials generated in somatosensory areas in the parietal cortex (Allison et al., 1991). This is likely due to anatomical (Pandya and Barnes, 1987) and functional (Yamaguchi and Knight, 1990) connectivity between the dorsolateral pre-frontal cortex and somatosensory areas. Yamaguchi and Knight (1990) showed that lesions of the DLPFC resulted in altered amplitude of early SEPs recorded from primary somatosensory cortex (S1) (Yamaguchi and Knight, 1990). The strong baseline phase connectivity between electrodes sites F3 and CP3 confirm this. As such, we hypothesized that transient inhibition of the left DLPFC from high field cTBS should alter SEP amplitudes measured from
S1 similar to the lesion results. This was indeed the case, and sham cTBS, which induced electric field amplitudes about 5\% of that produced by real TBS, trended toward the same effect. It is possible that low electric fields have weaker effects on downstream connected regions and may exert their effect mainly on local circuits within the maxima of the induced electric field. The SEP data from electrode site F3 support this. There was a significant difference in potential amplitude between sham stimulation and sham-sham stimulation only at site F3 which is supported by the phase connectivity results. Thus, there seems to be a graded response with respect to stimulation strength with strongest effects for real cTBS and weaker but not negligible effects for sham cTBS. Interestingly, the direct comparison of real versus sham stimulation did not result in significant phase synchrony effects for many channel pairs. This was not due to a 


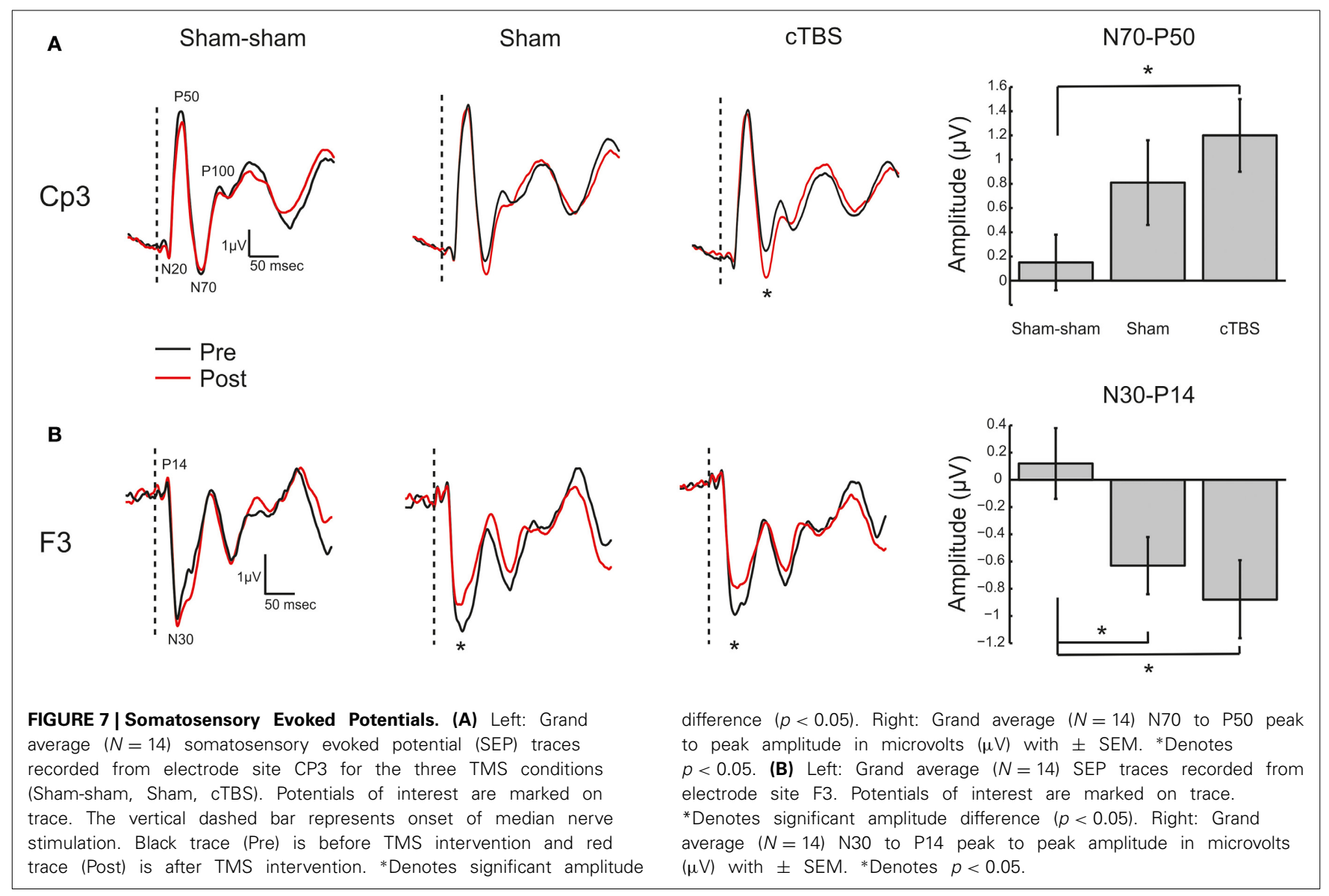

missing stimulation effect or non-specific effects due to habituation to the task over time. On the contrary, both real and sham cTBS showed stimulation induced changes in a similar direction compared to the sham-sham condition. The effect of weak electric fields delivered to DLPFC upon somatosensory processing is not unprecedented. Bolton and Staines (2011) also reported that their sham stimulation resulted in an effect upon parietal SEPs. We estimate the electric field induced by their sham condition (6\% resting motor threshold) (Bolton and Staines, 2011) induced an electric field in the left frontal cortex at around the same order of magnitude as that applied in this study. Thus, low electric field strength produced by sham stimulation exerts differential effects upon excitability in cortical areas directly under the TMS coil where the electric field is largest but also crucially can affect downstream anatomically and functionally connected regions. While not being the main focus of our study the effects of cTBS increased over time and we subsequently selected the post stimulation timepoint after $25 \mathrm{~min}$ to evaluate the effects of real and sham stimulation. Stronger stimulation effects are not uncommon in brain stimulation protocols. For example in one study using tDCS to induce plasticity in the motor cortex strongest effects on motor evoked potentials have been found after $30 \mathrm{~min}$ (Kuo et al., 2013). Also in the original paper introducing theta burst stimulation (TBS) (Huang et al., 2005) there were larger effects or effects at least as strong after 20 min compared to the immediate after effect of the stimulation.
Studies of slight TMS intensity differences also report correspondingly varying effects: cTBS of the motor cortex at $65 \%$ of resting motor threshold decreased motor excitability while $70 \%$ increased it (Doeltgen and Ridding, 2011). Similar intensity dependent effects have been found for tACS using $140 \mathrm{~Hz}$ over M1 (Moliadze et al., 2012), such that $0.4 \mathrm{~mA}$ reduced the amplitude of MEPs while $1.0 \mathrm{~mA}$ enhanced them. Also, a reversal of stimulation effects from cathodal tDCS stimulation was found from inhibition to excitation by changing intensities from 1 to $2 \mathrm{~mA}$ (Batsikadze et al., 2013). A possible mechanism for a reversal in the direction of effects using different intensities might be that inhibitory neurons are more rapidly excitable than excitatory neurons by lower field strengths. For higher field strengths excitatory neurons are more strongly activated and prevail over inhibitory effects that lead to a net excitatory effect (Berger et al., 2011; Herrmann et al., 2013). We found differential effects on SEP amplitudes both at the site of stimulation and in S1 concomitant with differences in connectivity between F3 and CP3 for sham and real cTBS.

Sham approaches for TMS have been investigated and constantly improved over the last decade. Early sham approaches were implemented by tilting the TMS coil away from the head, which was shown to induce significant residual electric fields (Loo et al., 2000; Lisanby et al., 2001). For example tilting the TMS coil $90^{\circ}$ away from the head can still induce electric field strengths that are up to $30 \%$ compared to the standard coil position 
Table 1 | Somatosensory evoked potential amplitudes.

\begin{tabular}{|c|c|c|c|c|}
\hline & cTBS & Sham & sS & $P$-value \\
\hline N20-P14 & $-0.19(0.09)$ & $-0.10(0.07)$ & $0.06(0.07)$ & 0.22 \\
\hline N33-P27 & $-0.06(0.15)$ & $-0.01(0.10)$ & $0.02(0.12)$ & 0.95 \\
\hline P50-N33 & $-0.18(0.15)$ & $0.18(0.49)$ & $-0.10(0.21)$ & 0.79 \\
\hline N70-P50 & $1.20(0.30)$ & $0.81(0.35)$ & $0.15(0.23)$ & 0.0491 \\
\hline N140-P100 & $0.28(0.27)$ & $0.26(0.37)$ & $0.30(0.34)$ & 0.99 \\
\hline \multicolumn{5}{|l|}{ F3 } \\
\hline N30-P14 & $-0.88(0.29)$ & $-0.63(0.21)$ & $0.12(0.26)$ & 0.0258 \\
\hline P45-N30 & $-0.36(0.42)$ & $0.16(0.25)$ & $0.02(0.19)$ & 0.47 \\
\hline N60-P45 & $-0.32(0.33)$ & $0.19(0.24)$ & $0.02(0.13)$ & 0.36 \\
\hline P80-N60 & $-0.62(0.52)$ & $-0.46(0.33)$ & $-0.21(0.29)$ & 0.77 \\
\hline
\end{tabular}

Group average (mean and SEM, N = 14) peak-to-peak amplitudes in $\mu$ V from channel CP3 (top) and F3 (bottom). cTBS, real stimulation; Sham, weak field stimulation; SS, sham-sham.

Bold values highlight significant effects (p-value $<0.05)$ in the Anova.

(Figure 8C). Current sham approaches are much more sophisticated, using a combined real or sham coil (Rossi et al., 2007) that can be electronically switched (Deng and Peterchev, 2011), concomitant audio stimulation and surface electrodes attached to the head that deliver electric current in unison with TMS pulsing to help ensure that skin sensations are identical between conditions. These approaches are very effective at disguising the appearance and accompanying sensory stimulation associated with TMS and are important features needed to investigate effects between real and sham stimulation only due to the direct effect of the induced electric field. However, in our opinion, not enough attention has been given to the residual electric field that is induced in the brain during sham treatments. While magnetic shields reduce the electric field effectively to about $5 \%$ of its strength during real stimulation, these fields are still in the range that has been demonstrated to induce lasting effects on neuronal populations. The sham TMS electric field is about an order of magnitude stronger than that produced by $1 \mathrm{~mA}$ tDCS (Figures 8A,B) that has proven to produce both cortical excitability and behavior changes in several studies (Fecteau et al., 2007; Holland et al., 2011; Ruff et al., 2013). Although the temporal course of the electric fields is usually different between electrical and magnetic stimulation methods and different protocols cannot easily be directly related to each other, this comparison shows that electric fields occurring during sham TMS are clearly within the range where physiological effects are possible. While the study of effects of weak electric fields induced by TMS is an interesting topic in itself, it is not desirable to have direct neuronal effects of sham stimulation during cognitive studies or especially clinical trials, since the sham condition could indeed be having an effect upon neuronal excitability. Thus the comparison of real and sham effects is made explicitly difficult especially in the case of missing effects between real and sham stimulation. Such observations taken at face value may lead one to conclude that a particular sham controlled TMS intervention was not effective when indeed they both were. It should be noted that this study employed two different subject groups such that possible between groups effects might interfere with our results. However, the baseline connectivity did not significantly differ between groups, as well as the analysis of pre-post changes that control for non-specific effects make this unlikely. These non-specific effects (for example anticipation of getting stimulated) may also contribute to effects between conditions though we were sensitive to keeping sensory stimuli (coil pressure on the head, auditory stimulation) the same during sham and sham-sham stimulation. Furthermore, all subjects in all conditions were naïve to TMS and did not know what to expect if anything across repeated sessions thus limiting the possibility that results were due to non-specific effects. Finally, these data are the result of a single commercial sham-coil using a cTBS rTMS protocol and may not directly transfer to other commercial sham-coils due to differences in the technology used to achieve shamming or to other rTMS protocols. Future studies should address these effects across other sham coils and TMS protocols.

\section{CONCLUSIONS}

Sham procedures are absolutely necessary to control for expectation effects which can manifest themselves on a physiological level (Büchel et al., 2014; Fiorio et al., 2014). It is clear that sham TMS, as employed here, produces electric fields in the human cortex of a magnitude that can affect cortical excitability. It is conceivable that the effect of real TMS stimulation may be underestimated in studies comparing it to current shamming protocols. A sham condition that mimics expectation and sensation but does not induce any electric field in the cortex is optimal for assessing effects of TMS, but, until this becomes common practice, it is our opinion that the weak electric field induced by current sham TMS approaches should be accounted for or at least considered when interpreting results. Nevertheless, we cannot readily generalize our findings for other rTMS protocols and future research has to investigate whether effects of weak TMS might occur for them as well. 


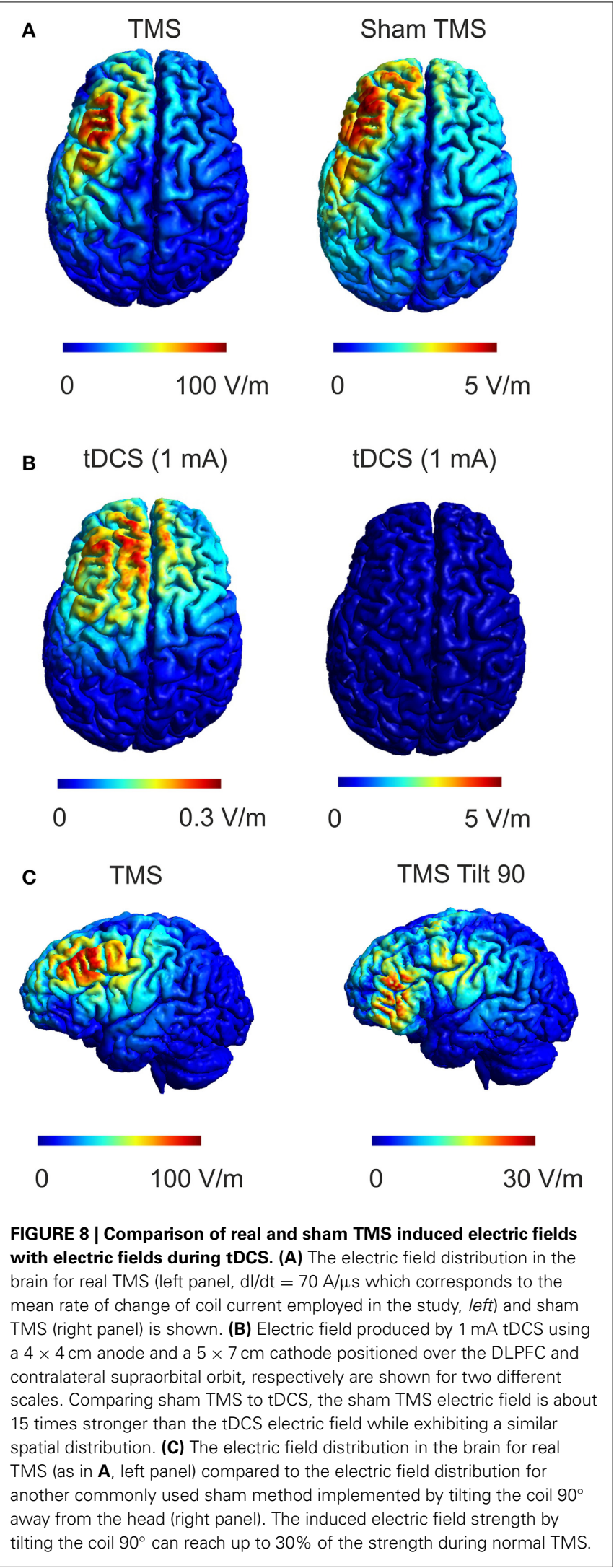

\section{ACKNOWLEDGMENTS}

The authors would like to thank Amanda Williams and Michael J. Wesley for help with data collection.

\section{SUPPLEMENTARY MATERIAL}

The Supplementary Material for this article can be found online at: http://www.frontiersin.org/journal/10.3389/fnhum. 2014.01043/abstract

\section{Supplementary Figure 1 | Buildup effect for changes in cortical EEG} connectivity after cTBS. (A) The weighted phase lag index (WPLI) for the F3-CP3 channel pair during median nerve stimulation is shown for three different timepoints (Pre, Post1, and Post2). The WPLI shows incremental increases in the high alpha, low beta range over the two post stimulation timepoints. (B) Connectivity changes Post1-Pre (left panel) and Post2-Pre (right panel) are shown to illustrate the effect of increased differences over time.

\section{REFERENCES}

Allison, T., McCarthy, G., Wood, C. C., and Jones, S. J. (1991). Potentials evoked in human and monkey cerebral cortex by stimulation of the median nerve. A review of scalp and intracranial recordings. Brain 114(Pt 6), 2465-2503.

Anastassiou, C. A., Perin, R., Markram, H., and Koch, C. (2011). Ephaptic coupling of cortical neurons. Nat. Neurosci. 14, 217-223. doi: 10.1038/nn.2727

Batsikadze, G., Moliadze, V., Paulus, W., Kuo, M. F., and Nitsche, M. A. (2013). Partially non-linear stimulation intensity-dependent effects of direct current stimulation on motor cortex excitability in humans. J. Physiol. 591, 1987-2000. doi: 10.1113/jphysiol.2012.249730

Berger, U., Korngreen, A., Bar-Gad, I., Friedman, A., Wolfus, S., Yeshurun, Y., et al. (2011). Magnetic stimulation intensity modulates motor inhibition. Neurosci. Lett. 504, 93-97. doi: 10.1016/j.neulet.2011.09.004

Bikson, M., Inoue, M., Akiyama, H., Deans, J. K., Fox, J. E., Miyakawa, H., et al. (2004). Effects of uniform extracellular DC electric fields on excitability in rat hippocampal slices in vitro. J. Physiol. 557, 175-190. doi: 10.1113/jphysiol.2003.055772

Bolton, D. A., and Staines, W. R. (2011). Transient inhibition of the dorsolateral prefrontal cortex disrupts attention-based modulation of tactile stimuli at early stages of somatosensory processing. Neuropsychologia 49, 1928-1937. doi: 10.1016/j.neuropsychologia.2011.03.020

Büchel, C., Geuter, S., Sprenger, C., and Eippert, F. (2014). Placebo analgesia: a predictive coding perspective. Neuron 81, 1223-1239. doi: 10.1016/j.neuron.2014.02.042

Datta, A., Bansal, V., Diaz, J., Patel, J., Reato, D., and Bikson, M. (2009). Gyri-precise head model of transcranial direct current stimulation: improved spatial focality using a ring electrode versus conventional rectangular pad. Brain Stimul. 2, 201-207, 207.e201. doi: 10.1016/j.brs.2009.03.005

Deans, J. K., Powell, A. D., and Jefferys, J. G. (2007). Sensitivity of coherent oscillations in rat hippocampus to AC electric fields. J. Physiol. 583, 555-565. doi: 10.1113/jphysiol.2007.137711

Delorme, A., and Makeig, S., (2004). EEGLAB: an open source toolbox for analysis of single-trial EEG dynamics including independent component analysis. $J$. Neurosci. Methods 134, 9-21. doi: 10.1016/j.jneumeth.2003.10.009

Deng, Z. D., and Peterchev, A. V. (2011). Transcranial magnetic stimulation coil with electronically switchable active and sham modes. Conf. Proc. IEEE Eng. Med. Biol. Soc. 2011, 1993-1996. doi: 10.1109/IEMBS.2011. 6090561

Dieckhofer, A., Waberski, T. D., Nitsche, M., Paulus, W., Buchner, H., and Gobbele, R. (2006). Transcranial direct current stimulation applied over the somatosensory cortex - differential effect on low and high frequency SEPs. Clin. Neurophysiol. 117, 2221-2227. doi: 10.1016/j.clinph.2006.07.136

Doeltgen, S. H., and Ridding, M. C. (2011). Low-intensity, short-interval theta burst stimulation modulates excitatory but not inhibitory motor networks. Clin. Neurophysiol. 122, 1411-1416. doi: 10.1016/j.clinph.2010.12.034 
Duecker, F., and Sack, A. T. (2013). Pre-stimulus sham TMS facilitates target detection. PLoS ONE 8:e57765. doi: 10.1371/journal.pone.0057765

Fecteau, S., Knoch, D., Fregni, F., Sultani, N., Boggio, P., and Pascual-Leone, A. (2007). Diminishing risk-taking behavior by modulating activity in the prefrontal cortex: a direct current stimulation study. J. Neurosci. 27, 12500-12505. doi: 10.1523/JNEUROSCI.3283-07.2007

Feurra, M., Paulus, W., Walsh, V., and Kanai, R. (2011). Frequency specific modulation of human somatosensory cortex. Front. Psychol. 2:13. doi: 10.3389/fpsyg.2011.00013

Fiorio, M., Emadi Andani, M., Marotta, A., Classen, J., and Tinazzi, M. (2014). Placebo-Induced changes in excitatory and inhibitory corticospinal circuits during motor performance. J. Neurosci. 34, 3993-4005. doi: 10.1523/JNEUROSCI.3931-13.2014

Francis, J. T., Gluckman, B. J., and Schiff, S. J. (2003). Sensitivity of neurons to weak electric fields. J. Neurosci. 23, 7255-7261.

Frohlich, F., and McCormick, D. A. (2010). Endogenous electric fields may guide neocortical network activity. Neuron 67, 129-143. doi: 10.1016/j.neuron.2010.06.005

Herrmann, C. S., Rach, S., Neuling, T., and Struber, D. (2013). Transcranial alternating current stimulation: a review of the underlying mechanisms and modulation of cognitive processes. Front. Hum. Neurosci. 7:279. doi: 10.3389/fnhum.2013.00279

Holland, R., Leff, A. P., Josephs, O., Galea, J. M., Desikan, M., Price, C. J., et al. (2011). Speech facilitation by left inferior frontal cortex stimulation. Curr. Biol. 21, 1403-1407. doi: 10.1016/j.cub.2011.07.021

Huang, Y. Z., Edwards, M. J., Rounis, E., Bhatia, K. P., and Rothwell, J. C. (2005). Theta burst stimulation of the human motor cortex. Neuron 45, 201-206. doi: 10.1016/j.neuron.2004.12.033

Kuo, H. I., Bikson, M., Datta, A., Minhas, P., Paulus, W., Kuo, M. F., et al. (2013). Comparing cortical plasticity induced by conventional and high-definition 4 x 1 ring tDCS: a neurophysiological study. Brain Stimul. 6, 644-648. doi: 10.1016/j.brs.2012.09.010

Lisanby, S. H., Gutman, D., Luber, B., Schroeder, C., and Sackeim, H. A. (2001). Sham TMS: intracerebral measurement of the induced electrical field and the induction of motor-evoked potentials. Biol. Psychiatry 49, 460-463. doi: 10.1016/S0006-3223(00)01110-0

Loo, C. K., Taylor, J. L., Gandevia, S. C., McDarmont, B. N., Mitchell, P. B., and Sachdev, P. S. (2000). Transcranial magnetic stimulation (TMS) in controlled treatment studies: are some "sham" forms active? Biol. Psychiatry 47, 325-331. doi: 10.1016/S0006-3223(99)00285-1

Maris, E., and Oostenveld, R., (2007). Nonparametric statistical testing of EEG- and MEG-data. J. Neurosci. Methods 164, 177-190. doi: 10.1016/j.jneumeth.2007.03.024

Matsunaga, K., Nitsche, M. A., Tsuji, S., and Rothwell, J. C. (2004). Effect of transcranial DC sensorimotor cortex stimulation on somatosensory evoked potentials in humans. Clin. Neurophysiol. 115, 456-460. doi: 10.1016/S13882457(03)00362-6

Miranda, P. C., Correia, L., Salvador, R., and Basser, P. J. (2007). Tissue heterogeneity as a mechanism for localized neural stimulation by applied electric fields. Phys. Med. Biol. 52, 5603-5617. doi: 10.1088/0031-9155/52/18/009

Miranda, P. C., Mekonnen, A., Salvador, R., and Ruffini, G. (2013). The electric field in the cortex during transcranial current stimulation. Neuroimage 70, 48-58. doi: 10.1016/j.neuroimage.2012.12.034

Moliadze, V., Antal, A., and Paulus, W. (2010). Boosting brain excitability by transcranial high frequency stimulation in the ripple range. J. Physiol. 588, 4891-4904. doi: 10.1113/jphysiol.2010.196998

Moliadze, V., Atalay, D., Antal, A., and Paulus, W. (2012). Close to threshold transcranial electrical stimulation preferentially activates inhibitory networks before switching to excitation with higher intensities. Brain Stimul. 5, 505-511. doi: 10.1016/j.brs.2011.11.004

Nitsche, M. A., and Paulus, W. (2000). Excitability changes induced in the human motor cortex by weak transcranial direct current stimulation. J. Physiol. $527(\mathrm{Pt}$ 3), 633-639. doi: 10.1111/j.1469-7793.2000.t01-1-00633.x
Opitz, A., Legon, W., Rowlands, A., Bickel, W. K., Paulus, W., and Tyler, W. J. (2013). Physiological observations validate finite element models for estimating subject-specific electric field distributions induced by transcranial magnetic stimulation of the human motor cortex. Neuroimage 81, 253-264. doi: 10.1016/j.neuroimage.2013.04.067

Opitz, A., Windhoff, M., Heidemann, R. M., Turner, R., and Thielscher, A. (2011). How the brain tissue shapes the electric field induced by transcranial magnetic stimulation. Neuroimage 58, 849-859. doi: 10.1016/j.neuroimage.2011.06.069

Ozen, S., Sirota, A., Belluscio, M. A., Anastassiou, C. A., Stark, E., Koch, C., et al. (2010). Transcranial electric stimulation entrains cortical neuronal populations in rats. J. Neurosci. 30, 11476-11485. doi: 10.1523/JNEUROSCI.5252-09.2010

Pandya, D. N., and Barnes, C. L. (1987). "Architecture and connections of the frontal lobe," in The Frontal Lobes Revisited, ed E. Perecman. (New York, NY: The IRBN Press), 41-72.

Radman, T., Su, Y., An, J. H., Parra, L. C., and Bikson, M. (2007). Spike timing amplifies the effect of electric fields on neurons: implications for endogenous field effects. J. Neurosci. 27, 3030-3036. doi: 10.1523/JNEUROSCI.0095-07.2007

Rossi, S., Ferro, M., Cincotta, M., Ulivelli, M., Bartalini, S., Miniussi, C., et al. (2007). A real electro-magnetic placebo (REMP) device for sham transcranial magnetic stimulation (TMS). Clin. Neurophysiol. 118, 709-716. doi: 10.1016/j.clinph.2006.11.005

Ruff, C. C., Ugazio, G., and Fehr, E. (2013). Changing social norm compliance with noninvasive brain stimulation. Science 342, 482-484. doi: 10.1126/science. 1241399

Salvador, R., Mekonnen, A., Ruffini, G., and Miranda, P. C. (2010). Modeling the electric field induced in a high resolution realistic head model during transcranial current stimulation. Conf. Proc. IEEE Eng. Med. Biol. Soc. 2010, 2073-2076. doi: 10.1109/IEMBS.2010.5626315

Salvador, R., Silva, S., Basser, P. J., and Miranda, P. C. (2011). Determining which mechanisms lead to activation in the motor cortex: a modeling study of transcranial magnetic stimulation using realistic stimulus waveforms and sulcal geometry. Clin. Neurophysiol. 122, 748-758. doi: 10.1016/j.clinph. 2010.09.022

Thielscher, A., and Kammer, T. (2004). Electric field properties of two commercial figure-8 coils in TMS: calculation of focality and efficiency. Clin. Neurophysiol. 115, 1697-1708. doi: 10.1016/j.clinph.2004.02.019

Vinck, M., Oostenveld, R., van Wingerden, M., Battaglia, F., and Pennartz, C. M. (2011). An improved index of phase-synchronization for electrophysiological data in the presence of volume-conduction, noise and sample-size bias. Neuroimage 55, 1548-1565. doi: 10.1016/j.neuroimage.2011.01.055

Windhoff, M., Opitz, A., and Thielscher, A. (2013). Electric field calculations in brain stimulation based on finite elements: an optimized processing pipeline for the generation and usage of accurate individual head models. Hum. Brain. Mapp. 34, 923-935. doi: 10.1002/hbm.21479

Yamaguchi, S., and Knight, R. T. (1990). Gating of somatosensory input by human prefrontal cortex. Brain Res. 521, 281-288. doi: 10.1016/0006-8993(90)91553-S

Conflict of Interest Statement: The authors declare that the research was conducted in the absence of any commercial or financial relationships that could be construed as a potential conflict of interest.

Received: 07 October 2014; accepted: 14 December 2014; published online: 08 January 2015.

Citation: Opitz A, Legon W, Mueller J, Barbour A, Paulus W and Tyler WJ (2015) Is sham cTBS real cTBS? The effect on EEG dynamics. Front. Hum. Neurosci. 8:1043. doi: 10.3389/fnhum.2014.01043

This article was submitted to the journal Frontiers in Human Neuroscience.

Copyright $\odot 2015$ Opitz, Legon, Mueller, Barbour, Paulus and Tyler. This is an openaccess article distributed under the terms of the Creative Commons Attribution License (CC BY). The use, distribution or reproduction in other forums is permitted, provided the original author $(s)$ or licensor are credited and that the original publication in this journal is cited, in accordance with accepted academic practice. No use, distribution or reproduction is permitted which does not comply with these terms. 